REVIEW: PHYSICAL SIGNS

The grasp and other primitive reflexes

J M Schott, M N Rossor

J Neurol Neurosurg Psychiatry 2003;74:558-560

Primitive reflexes are typically present in childhood, suppressed during normal development, and may reappear with diseases of the brain, particularly those affecting the frontal lobes. In this review we discuss some historical aspects surrounding these reflexes, how they might be elicited and interpreted, and their potential clinical utility in modern neurological practice.

$T$ he primitive reflexes are a group of behavioural motor responses which are found in normal early development, are subsequently inhibited, but may be released from inhibition by cerebral, usually frontal, damage. They are thus part of a broader group of reflexes which reflect release phenomena, such as exaggerated stretch reflexes and extensor plantars. They do however involve more complex motor responses than such simple stretch reflexes, and are often a normal feature in the neonate or infant.

References to primitive reflexes abound in textbooks of neurology, and they are often features of clinical demonstrations. However, they are often misinterpreted and their utility overemphasised in the modern era of detailed neuropsychology and neuroimaging. They can, however, be a valuable diagnostic adjunct to the examination in certain instances.

Many primitive reflexes are recognised. These include grasping, oral, nucocephalic, corneomandibular and glabella tap reflexes, and utilisation behaviour; these reflexes are discussed below. The palmomental reflex has recently been reviewed as part of this series. ${ }^{1}$

\section{GRASPING REFLEXES}

Grasping has been associated with lesions of the frontal lobes for nearly a century and referred to by a number of different terms including "the grasping reflex", ${ }^{2}$ "forced grasping" and "forced groping", ${ }^{3}$ the "instinctive grasp reaction", and "magnetic apraxia". ${ }^{5}$ Seyffarth and DennyBrown ${ }^{4}$ reviewed these reflexes in detail, and suggested their separation into two distinct responses: the grasp reflex and instinctive grasp reaction.

Eliciting the grasp reflex requires dual stimuli. The first is a distally moving deep pressure over a specific area of the palmar surface of the hand, which elicits a brief muscular contraction (the "catching phase"). This then develops into a strong "holding" phase only if traction is made on the tendons of the flexor or adductor muscles now in contraction, the response being maintained by continued traction. This "holding" phase can be distinguished from the myotactic resistance see in spasticity in that the former can be induced with any length of the finger flexors and is self sustaining. ${ }^{4}$ The grasp reflex may diminish if the dorsum of the hand is stroked, and thus the reflex may not be present if the patient's hand (palm uppermost) is rested in the examiner's palm. Opposition of the thumb may also be noted, possibly dependent on the size and nature of the object that is grasped.

In the instinctive grasp reaction, deliberate progressive closure of the whole hand is made in a series of small movements on a stationary contact within the palm, terminating eventually in a complete grip. When well developed, this reaction will occur in response to stationary light stimulation in any part of the hand, and is often preceded by series of movements bringing the object closer to the centre of the palm. When the reaction is weak, it may only be provoked by light pressure to the radial part of the hollow of the hand. Removal of the object may lead to movements of pursuit or grasping, and movement of the object within the palm leads to tightening of the grip. Pressure on the palm rather than movement appears to be the most important feature when eliciting this reflex. ${ }^{4}$ Many of the phenomena of this reaction have been classified as "forced groping" or "forced grasping" by other authors, ${ }^{3}$ and there may be an overlap or coexistence with utilisation behaviour.

It has long been recognised that both these reflexes are present in newborn infants, disappear during normal development, and may reappear in disease states, suggesting that these responses are suppressed but not lost during maturation. While bilateral grasp reflexes are often seen with degenerative diseases or diffuse vascular disease affecting the frontal lobes, attempts to localise the lesions responsible for these responses more accurately have not been consistent. Early descriptions suggested that unilateral grasp responses were due to lesions of the contralateral frontal lobe, ${ }^{35}$ possibly as a result of failure of the frontal lobe to inhibit parietal lobe function. ${ }^{5}$ More recently lesions in the supplementary motor cortex and cingulate gyrus have been implicated in the aetiology of these responses, although there is no consensus as to which region is responsible for which response. ${ }^{67}$ Moreover, lesions of the frontal lobes may abolish these reflexes, ${ }^{8}$ and grasping is often bilateral, even with a unilateral lesion. Practically, there may be little value in differentiating the palmar grasp from the instinctive grasp reaction-the presence of either should increase the suspicion of an underlying organic brain disorder. In our experience, groping reactions are much less common, and are associated with more advanced disease states.

A group of foot reflexes allied to the palmar grasp were described by a number of authors 
from the 1930s onwards. Brain and Curran described the grasp reflex of the foot, ${ }^{9}$ Goldstein the tonic foot response, ${ }^{10}$ and Seyyfarth and Denny-Brown the grasp reflex of the toes. ${ }^{4}$ Although there are subtle variations in the responses described, all these authors conclude that stimulating the sole of the foot (particularly over the metatarsophalangeal joints using a distally moving object) leads to flexion and adduction movements of the toes, and that while the reflex is distinct from the Babinski response, the two reflexes may appear concurrently. As with the palmar grasp, this response is seen in infants, may reappear consequent to damage to the frontal lobe or its efferent connections, and may be particularly frequent with contralateral, medial frontal lobe damage. ${ }^{11}$ However, as Seyyfarth and Denny-Brown found no patients with grasp reflex of the toes in the absence of finger grasping, ${ }^{4}$ it is unlikely that testing for the presence of this reflex will give more diagnostic information than can be deduced from the presence or absence of palmar grasping.

\section{UTILISATION BEHAVIOUR}

Utilisation behaviour was first described in five patients with either unilateral or bilateral frontal lobe lesions by Lhermitte in $1983 .{ }^{12}$ He noted that these patients, when presented with utilitarian objects, were compelled to grasp and use them. Examples of this behaviour were patients who, when presented with a bottle of water and a glass, but no instructions, would unquestioningly and automatically fill the glass and drink from it. The same, automatic, unquestioning behaviour could be elicited on presentation of a hammer and nail; envelope and sheet of paper; cigarette and lighter; and cheese, knife, and bread. Normal subjects failed to perform these tasks even when the objects were placed directly in their hands. ${ }^{12}$ Shallice et al further refined the concept of utilisation behaviour, by classifying it into two forms. ${ }^{13}$ The first "induced" form, occurs when, as shown by Lhermitte, an object is placed in front of the patient. The second "incidental" form is observed when the patient is involved in other tasks, and shows such behaviour when his or her attention has not been drawn to the object in question. Assessment of utilisation behaviour thus should include both careful observation of the patient's behaviour (particularly with respect to interaction with the external environment), as well as attempts to elicit such responses. A particularly striking example may be seen when a patient is presented with a series of pairs of spectacles, which he proceeds to put on his nose, one on top of another.

Utilisation behaviour may be localised to damage to the inferior frontal lobes, as originally proposed by Lhermitte. ${ }^{12}{ }^{13}$ However, it may also be seen with certain subcortical lesions, and particularly the thalamus (see Eslinger for review ${ }^{14}$ ). Whether this behaviour therefore results from a lack of frontal inhibition releasing the parietal lobes which are dependent on visual and tactile stimulation from the outside world as proposed by Lhermitte, ${ }^{12}$ or from disruption of intrafrontal mechanisms is unclear. ${ }^{14}$ The aetiology of utilisation behaviour can include stroke, neoplasm, frontotemporal dementia, corticobasal degeneration, and Alzheimer's disease. ${ }^{14}$ In our experience, the presence of either spontaneous or induced utilisation behaviour is highly indicative of underlying organic brain disease.

\section{PRIMITIVE ORAL REFLEXES}

Despite being frequently cited and demonstrated, the primitive oral reflexes are often wrongly interpreted, in part as a result of confusing terminology. The true primitive oral reflexes include the sucking, rooting, and snout reflexes, which can all be considered as appropriate feeding responses in infants.
Sucking reflexes may be seen in response to tactile stimulation in the oral region, or in response to the insertion of an object (for example, a spatula) into the mouth. The snout reflex is present when the lips pucker in response to gentle pressure over the nasal philtrum. Rooting responses are seen when the mouth turns towards an object gently stroking the cheek (tactile rooting), or towards an object (for example, tendon hammer) brought into the patient's field of view (visual rooting). Particular care must be taken to distinguish these reflexes from tardive or buccolingual dyskinaesias in patients who have been exposed to neuroleptics.

By contrast, closure of the mouth with pouting of the lips elicited by either tapping around the mouth or onto the lips (or tapping a spatula placed over the lips) represents an increased myotactic stretch reflex, and not a primitive response. Pouting may be present (at least electromyographically) in normal individuals, ${ }^{15}$ although brisk responses, indicative of upper motor neurone lesions, may be seen in the context of amyotrophic lateral sclerosis, multiple sclerosis, or small vessel cerebrovascular disease. ${ }^{16}$ Confusingly, the terms snout and pout have historically often been used interchangeably. ${ }^{11}$ To avoid confusion, we suggest that the term pouting should only be used to describe the increased myotactic stretch reflex.

\section{GLABELLA TAP REFLEX}

In 1896 Overend first reported that in the normal subject, light tapping over the glabella produces a reflex blinking of both eyes. ${ }^{17}$ This reflex has been referred to as the glabella tap sign, nasopalpebral reflex, and blinking reflex. The reflex has two components; an initial myotactic monosynaptic reflex is followed by a later multisynaptic nociceptive reflex. In normal subjects the latter response habituates, showing increased latency and decreasing amplitude. ${ }^{18}$ A positive glabella tap reflex refers to failure of habituation of the response-that is, when repeated tapping continues to produce a blinking reaction. The reflex is thought to be similar to the reflex blepharospasm seen in newborn and premature infants, in whom it can continue for six months. ${ }^{19}$

The glabella tap has long been thought to be an early sign of Parkinson's disease; Garland, writing in 1952, stated that "... On tapping the glabella both eyelids blink in time with the tapping, whether this be fast or slow, and this blinking will continue indefinitely; the normal person will only blink after the first few taps. For all practical purposes this physical sign is diagnostic of Parkinson's disease ${ }^{\prime 2}{ }^{20}$ However, the glabella tap reflex may also be positive as a release phenomenon as a result of diffuse (probably frontal lobe) damage, ${ }^{21}$ and in one report was positive in $36 \%$ of patients with no intracranial pathology. ${ }^{18}$ As the glabella tap reflex is neither sensitive for the presence of intracerebral pathology, nor specific for parkinsonism, its role in modern clinical practice is questionable.

\section{CORNEOMANDIBULAR REFLEX (WARTENBERG'S REFLEX)}

A horizontal movement of the mandible to the contralateral side on touching the cornea of the ipsilateral eye was first described by von Sölder in 1902. ${ }^{22}$ This observation went largely unnoticed outside Germany until Wartenberg's extensive review of the "winking jaw phenomenon" in 1948. ${ }^{23}$ Wartenberg (who suggested that the reflex be elicited with some pressure to the cornea, best applied with a solid glass applicator) regarded this reflex as an inverted and reversed Marcus Gunn (or "jaw winking") phenomenon in which "... on lateral movement of the jaw to the right side (left external pterygoid), the left upper lid is raised quickly and powerfully . . .. ${ }^{24}$ Wartenberg suggested that these two phenomena were caused by an associated movement between the orbicularis oculi and the external pterygoid muscles, due 
to a supranuclear lesion of the trigeminal nerve. ${ }^{23}$ The reflex may be present in acute coma (especially if due to a structural lesion $^{25}$ ), cerebrovascular disease, multiple sclerosis, Parkinson's disease, and amyotrophic lateral sclerosis. ${ }^{26}$

\section{NUCHOCEPHALIC REFLEX}

The nuchocephalic reflex was first described by Jenkyn et al in this journal. ${ }^{27}$ It is elicited by rapidly turning the shoulders of a standing patient to the right or left. The reflex is present ("uninhibited") if the head holds its active original position through active contracture of the cervical muscles. In infants, the reflex is present ("uninhibited") until around the age of 4, when it disappears ("inhibited"). Jenkyn et al found the presence of the reflex in adults to be correlated directly with evidence of diffuse cerebral dysfunction, and concluded that is was a sensitive indicator of the status of higher cortical function. The reflex has not received widespread acceptance; a PubMed search reveals only one citation since the original description.

\section{PREVALENCE OF PRIMITIVE REFLEXES}

Brown et al examined 240 healthy young adults, and found that $3 \%$ had a sucking reflex, $1 \%$ a grasp, with none having a snout reflex. ${ }^{28}$ The prevalence of many of these reflexes may increase with age ${ }^{29}$ possibly in relation to an increased ischaemic burden. ${ }^{30}$ Paulson and Gottlieb examined 85 patients with "arteriosclerosis, presenile brain disease, or senile brain disease", in whom neoplasia was excluded. They reported that $53 \%$ had a sucking reflex, $52 \%$ a snout, $18 \%$ a grasp, and $7 \%$ a corneomandibular reflex. ${ }^{11}$ It seems likely that while the presence of no single primitive reflexes reliably detects frontal lobe pathology, the presence of some may be more useful that others. In our clinical practice, we place most weight on the presence of utilisation behaviour, oral reflexes, and asymmetric primitive reflexes. Multiple primitive reflexes in combination may be most predictive of organic (usually frontal lobe) pathology.

\section{CONCLUSION}

In the modern era, with detailed non-invasive imaging and neuropsychological testing widely available, the role of eliciting primitive reflexes may seem limited. The complexity of many of these responses makes it perhaps unsurprising that detailed anatomical localisation, despite the availability of structural and functional imaging, has in general not been possible. Furthermore, variation in techniques used to elicit these reflexes and reports of their presence in young healthy volunteers $^{28}$ further add to difficulties in assessing their significance in clinical practice.

Nonetheless, these reflexes may still be useful in certain clinical scenarios. In particular, the presence of primitive reflexes in an apathetic patient with normal brain imaging may be helpful in suggesting the presence of an organic neurodegenerative process, particularly affecting the frontal lobes, rather than a primary psychiatric disorder. The development of grasp responses in a patient known to have a frontal lobe tumour or infarct may be an early clue to the extension of that lesion, and may suggest that reimaging is warranted. Thomas suggested that primitive reflexes may also be useful in predicting long term cognitive dysfunction after head injury; the appearance of new demyelinating lesions in "clinically stable" patients with multiple sclerosis; or in suggesting the development of hydrocephalus or shunt occlusion following chronic meningitis. ${ }^{32}$ In all these cases particular care must be taken when eliciting and interpreting these reflexes; it is particularly important to distinguish them from upper motor neurone signs, with which some of these responses can be confused.

\section{Authors' affiliations}

J M Schott, Dementia Research Group, Institute of Neurology, University College, London, UK

M N Rossor, Dementia Research Group, Institute of Neurology,

University College, London; and Division of Neuroscience and

Psychological Medicine, Imperial College, London, UK

\section{REFERENCES}

1 Owen G, Mulley GP. The palmomental reflex: a useful clinical sign? J Neurol Neurosurg Psychiatry 2002;73:1 13-15

2 Wilson SAK, Walshe FMR. The phenomenon of "tonic innervation" and its relation to motor apraxia. Brain 1914;37:199-246.

3 Adie WJ, Critchley M. Forced grasping and groping. Brain 1927;50: 142-70

4 Seyffarth $\mathbf{H}$, Denny-Brown $D$. The grasp reflex and the instinctive grasp reaction. Brain 1948;71:109-83.

5 Denny-Brown D. Nature of apraxia. J Nerv Ment Dis 1958;126:9-32.

6 De Renzi E, Barbieri C. The incidence of the grasp reflex following hemispheric lesion and its relation to frontal damage. Brain 1992;115:293-313

7 Hashimoto R, Tanaka Y. Contribution of the supplementary motor area and anterior cingulate gyrus to pathological grasping phenomena. Eur Neurol 1998:40:151-8

8 Paulson GW. The neurological examination in dementia. In: Wells EC, ed. Dementia. Philadelphia: F A Davis Company, 1977:169-88.

9 Brain WR, Curran RD. The grasp reflex of the foot. Brain 1932; 40:347-56.

10 Goldstein K. The tonic foot response to stimulation of the sole: its physiological significance and diagnostic value. Brain 1938;61:269-83.

11 Paulson GW, Gottlieb G. Developmental reflexes: the reappearance of foetal and neonatal reflexes in aged patients. Brain 1968;91:37-52.

12 Lhermitte F. "Utilization behaviour" and its relation to lesions of the frontal lobes. Brain 1983;106:237-55.

13 Shallice T, Burgess PW, Schon F, et al. The origins of utilization behaviour. Brain 1989:112:1587-98.

14 Eslinger PJ. The anatomic basis of utilisation behaviour: as shift from frontal-parietal to intra-frontal mechanisms. Cortex 2002;38:273-6.

15 Ekbom KA, Jernelius B, Kugelberg E. Perioral reflexes. Neurology 1952;2:103-11.

16 Rossor MN. Snouting, pouting and rooting. Practical Neurology 2001;1:119-21.

17 Overend $\mathbf{W}$. Preliminary note on a new cranial reflex. Lancet 1896;1:619

18 Jensen JPA, Gron U, Pakkenberg H. Comparison of three primitive reflexes in neurological patients and in normal individuals. J Neurol Neurosurg Psychiatry 1983;46:162-7.

19 Fisher CM. Reflex blepharospasm. Neurology 1963;13:77-8.

20 Garland HG. Parkinsonism. BM 1952;1:153-5.

21 Pearce J, Aziz H, Hallagher JC. Primitive reflex activity in primary and symptomatic parkinsonism. J Neurol Neurosurg Psychiatry 1968;31:501-8.

22 von Sölder F. Der Corneo-Mandibularrefelx. Neurol Centralb 1902;21:111.

23 Wartenberg R. Winking-jaw phenomenon. Arch Neurol Psychiatry Chicago 1948;59:734-53.

24 Gunn RM. Congenital ptosis with peculiar associated movements of the affected lid. Trans Opthalmol Soc U K 1883;3:283.

25 Guberman A. Clinical significance of the corneomandibular reflex. Arch Neurol 1982;39:578-80.

26 Okuda B, Kodama N, Kawabata K, et al. Corneomandibular reflex in ALS. Neurology 1999:52:1699-701.

27 Jenkyn LR, Walsh DB, Walsh BT, et al. The nuchocephalic reflex. J Neurol Neurosurg Psychiatry 1975;38:561-6.

28 Brown DL, Smith TL, Knepper LE. Evaluation of five primitive reflexes in 240 young adults. Neurology 1998;51:322.

29 Jenkyn LR, Reeves AG, Warren T, et al. Neurologic signs in senescence. Arch Neurol 1985:42:1154-7.

30 Kobayashi S, Yamaguchi S, Okada K, et al. Primitive reflexes and MRI findings, cerebral blood flow in normal elderly. Gerontology 1990;36: 199-205.

31 Isakov E, Sazbon L, Costeff $\mathrm{H}$, et al. The diagnostic value of three common primitive reflexes. Eur Neurol 1984;23:17-21.

32 Thomas RJ. Blinking and the release reflexes: are they clinically useful? J Am Geriatr Soc 1994;42:609-13. 